\title{
ESTUDO QUÍMICO E BIOLÓGICO DE Tephrosia toxicaria Pers.
}

Jackson Nunes e Vasconcelos, Jefferson Queiroz Lima, Telma Leda Gomes de Lemos, Maria da Conceição Ferreira de Oliveira, Maria Mozarina Beserra Almeida, Manoel Andrade-Neto, Jair Mafezoli e Angela Martha Campos Arriaga*

Departamento de Química Orgânica e Inorgânica, Centro de Ciências, Universidade Federal do Ceará, CP 12200, 60021-970 Fortaleza - CE, Brasil

Gilvandete Maria Pinheiro Santiago

Departamento de Farmácia, Faculdade de Farmácia, Odontologia e Enfermagem, Universidade Federal do Ceará, Rua Capitão Francisco Pedro 1210, 60430-370 Fortaleza - CE, Brasil

Raimundo Braz-Filho\#

Departamento de Química Orgânica e Inorgânica, Universidade Federal do Ceará, Fortaleza - CE, Brasil

Recebido em 27/3/08; aceito em 23/9/08; publicado na web em 2/2/09

\begin{abstract}
CHEMICAL AND BIOLOGICAL STUDY OF THE Tephrosia toxicaria Pers. The ethanol extracts from leaves, stems, pods and roots were assayed against the $3^{\text {rd }}$ instar Aedes aegypti larvae and the highest activity was observed in the roots extracts $\left(\mathrm{LC}_{50} 47.86\right.$ ppm). This extract was submitted to partition with hexane, chloroform, ethyl acetate and methanol. The respective fractions were bioassayed and the best larvicidal activities were identified in the hexane $\left(\mathrm{LC}_{50} 23.99 \mathrm{ppm}\right)$ and chloroform $\left(\mathrm{LC}_{50} 13.80 \mathrm{ppm}\right)$ fractions. Antioxidant activity (DDPH method) was observed in the ethanol extract $\left(\mathrm{IC}_{50} 276 \mu \mathrm{g} / \mathrm{mL}\right.$ ) from roots of T. toxicaria. Fractions from this extract were also tested and the highest antioxidant activity $\left(\mathrm{IC}_{50} 89 \mu \mathrm{g} / \mathrm{mL}\right)$ was found in the methanol fraction. The flavonoids iso-obovatin (1), obovatin (2), 6a,12a-dehydro- $\beta$-toxicarol (3), 6a,12a-dehydro- $\alpha$-toxicarol (4) and $\alpha$-toxicarol (5) were isolated and bioassayed against $A$. aegypti. The flavonoid $\mathbf{5}$ showed the best larvicidal activity $\left(\mathrm{LC}_{50} 24.55 \mathrm{ppm}\right)$. The antioxidant activity of 2 was investigated and showed $\mathrm{IC}_{50} 3.370 \mu \mathrm{g} / \mathrm{mL}$. The antioxidant and larvicidal activities of Tephrosia toxicaria are reported for the first time.
\end{abstract}

Keywords: Tephrosia toxicaria; flavonoids; biological activity.

\section{INTRODUÇÃO}

O gênero Tephrosia, família Leguminoseae Papilionoideae (Fabaceae), possui aproximadamente trezentas espécies, distribuídas em regiões tropicais e subtropicais. ${ }^{1}$ Espécies deste gênero apresentam capacidade biogenética para produzir substâncias com grande diversidade estrutural, tais como esteróides, aminoácidos e flavonóides, incluindo os isoflavonóides rotenóides. ${ }^{2}$ Estes últimos são conhecidos por apresentarem as atividades ictiotóxica ${ }^{3}$ e larvicida. ${ }^{4}$ Outras atividades biológicas importantes, como anti-tumoral e antiviral, aparecem também descritas na literatura. ${ }^{5,6}$

Tephrosia toxicaria Pers., popularmente conhecida por "timbó de caiena" ou "anil", é utilizada para capturar peixes ${ }^{7}$ e o extrato aquoso de suas raízes é aplicado como defensivo agrícola em hortas orgânicas na localidade de Guaraciaba do Norte - CE. A literatura relata o isolamento e atividade anticâncer de compostos isolados de T. toxicaria ${ }^{8,9}$ bem como a composição química e atividade larvicida de seu óleo essencial. ${ }^{10}$

A busca por substitutos para os inseticidas sintéticos tem produzido muitos trabalhos científicos, contemplando inclusive a utilização de extratos ou constituintes ativos provenientes de plantas. ${ }^{10-15}$ Esta procura pode ser justificada pela fácil degradação destes constituintes, menor toxicidade ao homem e, conseqüentemente, representa uma alternativa mais segura para o meio

*e-mail: angelamcarriaga@yahoo.com.br

\#Pesquisador Visitante 1- CNPq/Programa de Pós-graduação em Química Orgânica ambiente. ${ }^{15}$ Estas observações, aliadas à crescente incidência da febre dengue - endêmica nas Américas, ${ }^{16}$ sendo diagnosticados de 50 a 100 milhões de casos por ano ${ }^{17}$ - nos estimularam adicionalmente para uma prospecção química em T. toxicaria, com o objetivo de encontrar compostos sucedâneos aos larvicidas sintéticos utilizados no combate ao Aedes aegypti, mosquito vetor desta doença.

Os radicais livres, produzidos pelo metabolismo intracelular como resultado regular das funções normais das células, são espécies químicas altamente reativas e o seu aumento excessivo, denominado stress oxidativo, pode danificar os lipídios, as proteínas e o DNA, o que estaria associado ao aparecimento de várias doenças, como por exemplo, câncer, declínio de sistema imunológico, catarata, deficiência orgânica do cérebro e mal de Alzheimer. ${ }^{18}$ Os flavonóides são relatados como antioxidantes por sua capacidade de capturar várias espécies oxidantes, como os radicais hidroxila ou peróxido. ${ }^{19-21} \mathrm{~A}$ busca por antioxidantes naturais, principalmente para utilização na indústria alimentícia, devido a sua baixa toxicidade quando comparados com os produtos sintéticos, como terc-butil-4-metoxifenol (BHA) ou 2,6-di-tercbutil-4-metilfenol (BHT) ${ }^{22}$ nos motivou a buscar novas fontes potenciais de antioxidantes derivados de plantas abundantes no Nordeste brasileiro.

Em continuidade ao estudo de espécies do gênero Tephrosia $a^{8,10,23}$ relatamos a composição química e as atividades larvicida e antioxidante de $T$. toxicaria. Os flavonóides iso-obovatina (1), obovatina (2), 6a,12a-desidro- $\beta$-toxicarol (3), 6a,12a-desidro- $\alpha$-toxicarol (4) e $\alpha$-toxicarol (5) foram isolados e $\mathbf{2 , 4}$ e 5, testados frente às larvas de A. aegypti. 


\section{PARTE EXPERIMENTAL}

\section{Procedimentos experimentais gerais}

Os espectros de absorção na região do infravermelho foram obtidos em espectrômetro Perkin Elmer, modelo FT-IR Espectrum 1000, utilizando pastilhas de $\mathrm{KBr}$ para análise das amostras, com freqüência medida em $\mathrm{cm}^{-1}$. Os espectros de $\mathrm{RMN}^{1} \mathrm{He} \mathrm{e}^{13} \mathrm{C}$, uni (1D) e bidimensionais (2D), foram obtidos em espectrômetro Bruker, modelo Avance DRX-500 (CENAUREMN-UFC), que opera na frequiência de $500 \mathrm{MHz}$ para hidrogênio $\left({ }^{1} \mathrm{H}\right)$ e $125 \mathrm{MHz}$ para carbono-13 $\left({ }^{13} \mathrm{C}\right)$, usando como solvente $\mathrm{CDCl}_{3}$. Para a análise da atividade antioxidante, utilizou-se espectrofotômetro modelo B582 da marca Micronal. Os pontos de fusão, não corrigidos, foram determinados em aparelho Mettler Toledo, com placa aquecedora modelo FP82HT e central de processamento FP90, acoplada a microscópio óptico monocular. As determinações foram realizadas a uma velocidade de aquecimento de $5^{\circ} \mathrm{C} / \mathrm{min}$. Nas cromatografias de adsorção em coluna foram utilizadas gel de sílica 60 ( $\Phi$ 63-200 $\mu \mathrm{m})$ e gel de sílica 60 para cromatografia flash ( $\Phi 40-63 \mu \mathrm{m})$ da Merck. Os tamanhos das colunas (comprimento e diâmetro) variaram de acordo com as quantidades de amostras a serem cromatografadas e adsorventes empregados. Para as cromatografias em camada delgada (CCD) utilizou-se gel de sílica 60 (Ф 2-25 $\mu \mathrm{m})$ da Carlo Erba e cromatofolhas de plástico de gel de sílica $60 \mathrm{~F}_{254}$, da Merck. As revelações das substâncias foram realizadas através da exposição à irradiação ultravioleta (UV) em dois comprimentos de onda ( 254 e $365 \mathrm{~nm}$ ) e/ou por aspersão em solução ácida de vanilina seguida de aquecimento a aproximadamente $150{ }^{\circ} \mathrm{C}$.

\section{Material vegetal}

A espécie estudada foi coletada no município de Guaraciaba do Norte-CE, em setembro de 2003. O Prof. E. P. Nunes, Departamento de Biologia, Universidade Federal do Ceará (UFC), Fortaleza, CE, identificou a espécie como Tephrosia toxicaria Pers. e uma exsicata (número de registro 32.139) encontra-se depositada no Herbário Prisco Bezerra/UFC.

\section{Extração e isolamento dos constituintes químicos}

Folhas (443,0 g), talos (550,0 g), vagens (80,0 g) e raízes (109,0 g) foram separados, secos, moídos e submetidos à extração com etanol à temperatura ambiente. As soluções etanólicas obtidas foram concentradas sob baixa pressão, em aparelho evaporador rotativo, produzindo 16,0 g (TTFE), 26,0 g (TTTE), 11,0 g (TTVE) e 16,0 g (TTRE) de extrato, respectivamente. TTRE $(16,0 \mathrm{~g})$ foi adsorvido em gel de sílica $(20,0 \mathrm{~g})$ e submetido a fracionamento cromatográfico em funil de vidro sinterizado $(\Phi=10 \mathrm{~cm})$ utilizando como eluente $1,0 \mathrm{~L}$ de hexano, $\mathrm{CHCl}_{3}$, AcOEt e $\mathrm{MeOH}$, obtendo-se as frações TTREH $(0,9 \mathrm{~g})$, TTREC $(6,7 \mathrm{~g})$, TTREA $(1,0 \mathrm{~g})$ e TTREM $(5,7 \mathrm{~g})$, respectivamente.

Uma alíquota da fração TTREH (370 mg) foi submetida a uma cromatografia flash utilizando gradiente de eluição composto por hexano/ $\mathrm{CHCl}_{3}$. Foram coletadas frações de aproximadamente 10 $\mathrm{mL}$, que foram reunidas em grupos com base na semelhança revelada pela análise por CCD. A fração $(89,0 \mathrm{mg})$ eluída com hexano/ $\mathrm{CHCl}_{3}$ (1:1) foi submetida a nova separação cromatográfica em gel de sílica, usando hexano/ $\mathrm{CHCl}_{3}(6: 4)$ como eluente, e forneceu $5,4 \mathrm{mg}$ da iso-obovatina (1, 5-hidroxi-6",6"-dimetilcromeno-[2",3":7,6]flavanona). Parte da fração TTREC $(3,0 \mathrm{~g})$ foi cromatografada em gel de sílica, utilizando-se hexano/AcOEt (9:1) como eluente, e as frações recolhidas foram reunidas em quatro grupos (G-1 a G-4) após análise por CCD. O grupo G-1 foi identificado como a obovatina
(2, 5-hidroxi-6",6"-dimetilcromeno-[2",3":7,8]-flavanona, 57,7 $\mathrm{mg})$. O grupo G-2 (48,2 mg) foi recromatografado em gel de sílica e a eluição com $\mathrm{CH}_{2} \mathrm{Cl}_{2}$ forneceu o rotenóide 3 (6a,12a-desidro- $\beta$ toxicarol, 5,0 mg). O flavonóide 4 (6a,12a-desidro- $\alpha$-toxicarol, 17,6 $\mathrm{mg}$ ) foi isolado do grupo G-3 por cromatografia flash, utilizando como eluente $\mathrm{CH}_{2} \mathrm{Cl}_{2}$. O grupo G-4 $(141,0 \mathrm{mg})$ foi submetido à cromatografia isocrática em gel de sílica e eluição com $\mathrm{CH}_{2} \mathrm{Cl}_{2}$ e forneceu o $\alpha$-toxicarol $(\mathbf{5}, 18,9 \mathrm{mg})$.

\section{5-hidroxi-6",6"-dimetilcromeno-[2",3":7,6]-flavanona (1)}

Sólido amorfo amarelo; RMN de ${ }^{1} \mathrm{H}\left(500 \mathrm{MHz}, \mathrm{CDCl}_{3}\right)-\delta_{\mathrm{H}}$ (multiplicidade, $J$ em Hz): 12,30 (s, OH); 7,40-7,45 (m, H-2'-6'); 6,63 (d, 10, H-4"); 5,99 (s, H-8); 5,51 (d, 10, H-5"); 5,42 (dd, 13,0 e 3,0, H-2); 3,08 (dd, 17,1 e 13,0, H-3 ); 2,83 (dd, 17,1 e 3,0, H-3 ); 1,45 (s, H-8") e 1,44 (s, H-7"'). RMN de ${ }^{13} \mathrm{C}\left(125 \mathrm{MHz}, \mathrm{CDCl}_{3}\right)$ - $\delta_{\mathrm{C}}: 196,0$ (C-4); 162,6 (C-9); 162,2 (C-7); 158,7 (C-5); 138,6 (C1'); 129,1 (C-3'-5'); 126,5 (C-5”); 126,4 (C-2', 6'); 115,5 (C-4”); 103,4 (C-6, 10); 96,5 (C-8); 79,4 (C-2); 78,6 (C-6"); 43,6 (C-3) e 28,7 (C-7", 8").

\section{5-hidroxi-6",6"-dimetilcromeno-[2",3":7,8]-flavanona (2)}

Sólido cristalino amarelo; p.f.: $119,8-121,2^{\circ} \mathrm{C}$; RMN de ${ }^{1} \mathrm{H}(500$ $\left.\mathrm{MHz}, \mathrm{CDCl}_{3}\right)-\delta_{\mathrm{H}}($ multiplicidade, $J$ em Hz): 12,11 (s, OH); 7,40-7,48 (m, H-2'-6'); 6,56 (d, 10, H-4"'); 6,02 (s, H-6); 5,48 (d, 10, H-5"); 5,44 (dd, 13,0 e 3,0, H-2); 3,06 (dd, 17,1 e 13,0, H-3 ${ }_{\mathrm{ax}}$ ); 2,84 (dd, 17,1 e $3,0, \mathrm{H}_{-}{ }_{\text {eq }}$ ); 1,46 (s, H- 8 ") e 1,44 (s, H-7"'). RMN de ${ }^{13} \mathrm{C}(125$ $\left.\mathrm{MHz}, \mathrm{CDCl}_{3}\right)-\delta_{\mathrm{C}}: 195,9(\mathrm{C}-4) ; 164,0(\mathrm{C}-5) ; 162,5$ (C-7); 157,0 (C9); 138,7 (C-1'); 129,0 (C-3', 5'); 126,7 (C-5'); 126,2 (C-2', 6'); 115,8 (C-4"); 103,1 (C-10); 102,2 (C-8); 97,9 (C-6); 79,3 (C-2); 78,4 (C-6"); 43,5 (C-3); 28,7 (C-8") e 28,5 (C-7").

\section{6a,12a-desidro- $\beta$-toxicarol (3)}

Sólido amorfo amarelo; p.f.: 137,8-140,2 ${ }^{\circ} \mathrm{C}$; RMN de ${ }^{1} \mathrm{H}(500$ $\mathrm{MHz}, \mathrm{CDCl}_{3}$ ) - $\delta_{\mathrm{H}}$ (multiplicidade, $J$ em Hz): 13,23 (s, OH); 8,28 (s, H-1); 6,74 (d, 10, H-4'); 6,57 (s, H-4); 6,31 (s, H-8); 5,63 (d, 10, H-5'); 4,97 (s, H-6); 3,96 (s, $\left.\mathrm{OCH}_{3}-3\right)$; 3,88 (s, $\left.\mathrm{OCH}_{3}-2\right)$ e 1,48 (s, H-7', 8'). $\mathrm{RMN} \mathrm{de}{ }^{13} \mathrm{C}\left(125 \mathrm{MHz}, \mathrm{CDCl}_{3}\right)-\delta_{\mathrm{C}}: 179,5$ (C-12); 159,5 (C-9); 157,3 (C-6a, 11); 156,2 (C-7a); 149,4 (C-3); 146,5 (C-4a); 144,4 (C-2); 128,4 (C-5'); 115,7 (C-4'); 111,0 (C-12a); 110,2 (C-1); 110,1 (C-1a); 106,3 (C-11a); 106,1 (C-10); 100,8 (C-4); 95,1 (C-8); 78,3 (C-6'); 65,0 (C-6); 56,7 $\left(\mathrm{OCH}_{3}-3\right)$; 56,2 $\left(\mathrm{OCH}_{3}-2\right)$ e $28,5(\mathrm{C}-7$ ', 8 ').

\section{6a,12a-desidro- $\alpha$-toxicarol (4)}

Sólido amorfo amarelo; p.f.: $219,8-221,6{ }^{\circ} \mathrm{C}$; RMN de ${ }^{1} \mathrm{H}(500$ $\mathrm{MHz}, \mathrm{CDCl}_{3}$ ) $-\delta_{\mathrm{H}}$ (multiplicidade, $J$ em Hz): 12,99 (s, OH); 8,26 (s, H-1); 6,62 (d, 10, H-4'); 6,54 (s, H-4); 6,28 (s, H-10); 5,59 (d, 10, H-5'); 4,98 (s, H-6); 3,94 (s, $\left.\mathrm{OCH}_{3}-3\right)$; 3,87 (s, $\left.\mathrm{OCH}_{3}-2\right)$ e 1,47 (s, H-7', 8'). RMN de ${ }^{13} \mathrm{C}\left(125 \mathrm{MHz}, \mathrm{CDCl}_{3}\right)-\delta_{\mathrm{C}}: 179,5$ (C-12); 162,6 (C-11); 159,5 (C-9); 157,0 (C-6a); 151,1 (C-7a); 149,5 (C-3); 146,5 (C-4a); 144,4 (C-2); 127,9 (C-5'); 114,6 (C-4'); 111,0 (C-12a); 110,2 (C-1); 109,9 (C-1a); 106,2 (C-11a); 101,3 (C-8); 100,8 (C-4); 100,7 (C-10); 78,3 (C-6'); 65,0 (C-6); 56,6 (OCH -3$) ; 56,1(\mathrm{OCH} 3-2)$ e 28,4 (C-7', 8')

\section{$\alpha$-toxicarol (5)}

Sólido amorfo amarelo; p.f.: $192,6-194,8{ }^{\circ} \mathrm{C}$; RMN de ${ }^{1} \mathrm{H}(500$ $\left.\mathrm{MHz}, \mathrm{CDCl}_{3}\right)-\delta_{\mathrm{H}}$ (multiplicidade, $J$ em Hz): 12,19 (s, OH); 6,87 (s, H-1); 6,56 (d, 10, H-4'); 6,46 (s, H-4); 5,96 (s, H-10); 5,47 (d, 10, H-5'); 4,87 (t, 3,1, H-6a); 4,62 (dd, 12,1 e 3,1, H-6 ${ }_{\mathrm{ax}}$ ); 4,18 (d, 12,1, $\mathrm{H}-6_{\mathrm{eq}}$ ); 3,85 (d, 4,0, H-12a); 3,82 (s, $\left.\mathrm{OCH}_{3}-3\right) ; 3,80$ (s, $\left.\mathrm{OCH}_{3}-2\right)$; 1,44 (s, H-8') e 1,39 (s, H-7'). RMN de ${ }^{13} \mathrm{C}\left(125 \mathrm{MHz}, \mathrm{CDCl}_{3}\right)-\delta_{\mathrm{C}}$ : 194,4 (C-12); 164, 8 (C-11); 163,0 (C-9); 156,1 (C-7a); 149,9 (C-3); 147,5 (C-4a); 144,2 (C-2); 126,6 (C-5'); 115,6 (C-4'); 110,6 (C-1); 
104,7 (C-1a); 102,0 (C-8); 101,4 (C-11a); 101,3 (C-4); 98,0 (C-10); 78,5 (C-6'); 72,1 (C-6a); 66,2 (C-6); 56,6 ( $\left.\mathrm{OCH}_{3}-2\right)$; 56,1 ( $\left.\mathrm{OCH}_{3}-3\right)$; 43,8 (C-12a); 28,8 (C-8') e 28,5 (C-7').

\section{Atividade larvicida}

A atividade larvicida sobre Aedes aegypti no estágio III $^{24}$ foi medida usando a metodologia de Oliveira et al.. ${ }^{25}$ Alíquotas das amostras (1 a $10 \mathrm{mg}$ ) foram dissolvidas em 0,3 mL de DMSO e transferidas para um béquer, onde foram adicionadas 50 larvas juntamente com 19,7 mL de água. ${ }^{25}$ Após 24 h, foi realizada a contagem do número de larvas mortas. Os ensaios foram realizados em triplicata e os resultados mostrados em concentração (ppm) da amostra capaz de matar $50 \%$ das larvas $\left(\mathrm{CL}_{50}\right)$. Amostras que apresentaram valores para $\mathrm{CL}_{50}<100 \mathrm{ppm}$ foram consideradas ativas. ${ }^{26}$ Paralelamente foram realizados testes em branco, utilizando água e DMSO a 1,5\% e como padrão positivo o Temephos ${ }^{\circledR}$.

\section{Atividade antioxidante}

A atividade antioxidante dos extratos etanólicos das folhas, talos, raízes e vagens foi determinada com base na capacidade de seqüestro de radicais livres, utilizando o DPPH (2,2-difenil-1-picril-hidrazila). ${ }^{27,28}$ Adicionou-se em uma cubeta $1 \mathrm{~mL}$ de DPPH (60 mmol/L), 1,0 $\mathrm{mL}$ de etanol e realizou-se leitura, após $30 \mathrm{~min}$, em espectrofotômetro UV-VIS com absorbância de $520 \mathrm{~nm}$, empregando o etanol como branco. Uma solução de DPPH, sem adição das amostras, foi utilizada como controle. Em seguida, foi feita uma leitura testando as amostras em diferentes concentrações $\left(1,0 ; 0,5\right.$ e $\left.0,25 \mathrm{mg} \mathrm{mL}^{-1}\right)$. O cálculo da atividade antioxidante foi feito utilizando o programa GraphPad Prism 4 for Windows e no cálculo da $\mathrm{CI}_{50}$ utilizou-se a equação dose-resposta sigmoidal. $\mathrm{O}$ teste foi realizado em triplicata, tendo sido utilizado como padrões o Trolox, a vitamina $\mathrm{C}$ e o BHT. Os resultados foram expressos como $\mathrm{CI}_{50}$, que corresponde à concentração da amostra necessária para seqüestrar $50 \%$ de radicais livres.

\section{RESULTADOS E DISCUSSÃO}

O estudo químico de Tephrosia toxicaria Pers. permitiu o isolamento e a identificação das flavanonas iso-obovatina (1), ${ }^{29}$ inédita no gênero, e obovatina (2), ${ }^{30}$ e dos rotenóides, 6a,12a-desidro- $\beta$-toxicarol (3), ${ }^{31}$ 6a, 12a-desidro- $\alpha$-toxicarol (4) ${ }^{31}$ e $\alpha$-toxicarol (5) ${ }^{31}$ (Figura 1). As substâncias isoladas já se encontram registradas na literatura e tiveram suas identidades estruturais elucidadas definitivamente através da aplicação de métodos espectroscópicos, tais como IV e, principalmente, RMN ${ }^{1} \mathrm{H}$ e RMN ${ }^{13} \mathrm{C}$ [1D e 2D: correlação homonuclear de átomos de hidrogênios ${ }^{1} \mathrm{H}-{ }^{1} \mathrm{H}-\mathrm{COSY}$ e heteronuclear de átomos de hidrogênio e carbono através de uma ligação $\left({ }^{1} \mathrm{H}-{ }^{13} \mathrm{C}\right.$ COSY $\left.-{ }^{1} J_{\mathrm{CH}}, \mathrm{HMQC}\right)$ e a longa distância $\left({ }^{1} \mathrm{H}^{-13} \mathrm{C}-\mathrm{COSY}-{ }^{\mathrm{n}} J_{\mathrm{CH}}, \mathrm{n}=2\right.$ e $n=3, H M B C)]$. A confirmação das estruturas deduzidas com base na análise de dados espectrais envolveu também a comparação com dados descritos na literatura. ${ }^{29-31}$

A Tabela 1 mostra os resultados dos testes de atividade larvicida de extratos, frações e substâncias obtidas de T. toxicaria frente ao Aedes aegypti. Dentre os extratos testados, verificou-se que o extrato das raízes (TTRE) foi o único que apresentou resultado significativo $\left(\mathrm{CL}_{50} 47,86 \pm 2,75 \mathrm{ppm}\right)$, de acordo com Cheng et al..${ }^{26}$ Deste extrato foram obtidas as frações hexano (TTREH), clorofórmio (TTREC), acetato de etila (TTREA) e metanol (TTREM). Entretanto, somente as frações hexano e clorofórmio apresentaram alto potencial larvicida com $\mathrm{CL}_{50} 23,99 \pm 1,05$ ppm e $\mathrm{CL}_{50} 13,80 \pm 0,80$ ppm, respectivamente. Da fração hexano foi isolada a substância 1 . Da fração clorofórmio foram isoladas as substâncias 2-5. Em virtude das pequenas quantidades
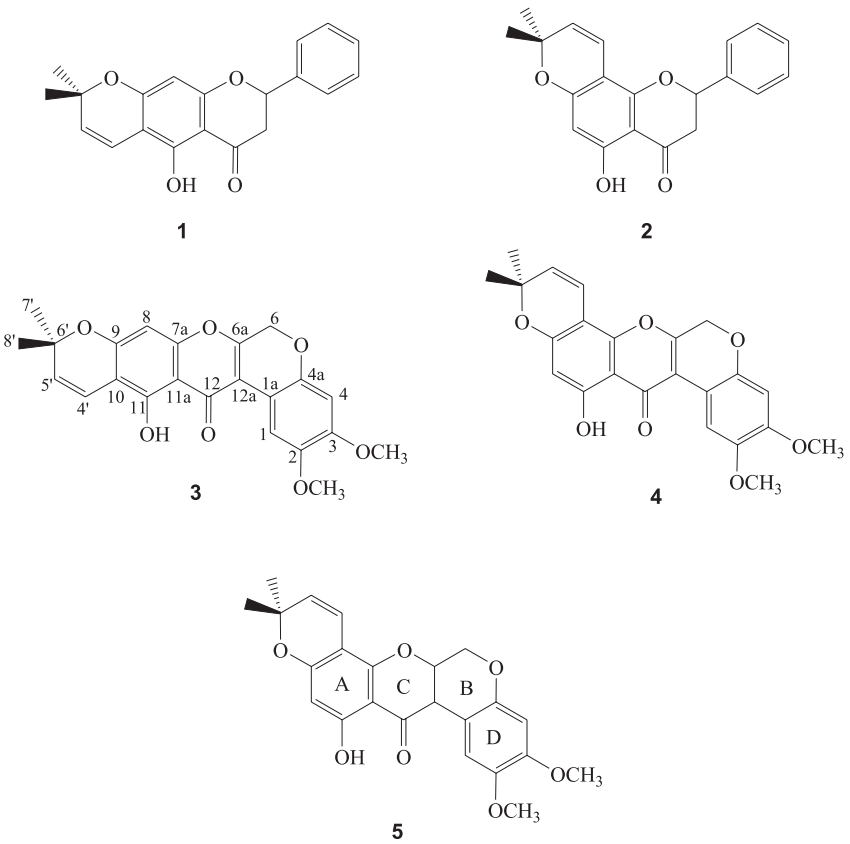

Figura 1. Flavonóides isolados do extrato etanólico das raízes de T. toxicaria Pers

isoladas, as substâncias $\mathbf{1}$ e $\mathbf{3}$ não foram testadas. As substâncias $\mathbf{2}$ e $\mathbf{4}$ apresentaram $\mathrm{CL}_{50}$ superiores a $50 \mathrm{ppm}$. A ausência da dupla ligação entre C-6a e C-12a, vista no $\alpha$-toxicarol (5) que apresentou $\mathrm{CL}_{50}$ de $24,55 \pm 0,13 \mathrm{ppm}$, pode justificar, segundo relatos da literatura, ${ }^{32} \mathrm{a}$ maior atividade deste composto quando comparado com os outros rotenóides, nos quais a junção entre os anéis B/C é insaturada. A maior atividade observada na avaliação do extrato clorofórmio pode ser justificada pela presença do $\alpha$-toxicarol e/ou por sinergismo com outras substâncias ativas não isoladas do extrato.

Tabela 1. Resultados de avaliação larvicida frente ao Aedes aegypti de extratos, frações e substâncias isoladas de T. toxicaria

\begin{tabular}{lc}
\hline Extrato & $\mathrm{CL}_{50}(\mathrm{ppm})$ \\
\hline TTFE & $218,78 \pm 1,50$ \\
TTTE & $275,90 \pm 1,25$ \\
TTVE & $115,22 \pm 1,38$ \\
TTRE & $47,86 \pm 2,75$ \\
\hline Fração & $\mathrm{CL}_{50}(\mathrm{ppm})$ \\
\hline TTREH & $23,99 \pm 1,05$ \\
TTREC & $13,80 \pm 0,80$ \\
TTREA & $95,50 \pm 1,48$ \\
TTREM & $>500$ \\
\hline Substâncias isoladas & $\mathrm{CL}_{50}(\mathrm{ppm})$ \\
\hline $\mathbf{1}$ & $\mathrm{NT}$ \\
$\mathbf{2}$ & $>50$ \\
$\mathbf{3}$ & $\mathrm{NT}$ \\
$\mathbf{4}$ & $>50$ \\
$\mathbf{5}$ & $24,55 \pm 0,13$ \\
\hline Padrão & $\mathrm{CL}_{50}(\mathrm{ppm})$ \\
\hline Temephos ${ }^{\circledR}$ & $1,40 \pm 0,20$ \\
\hline
\end{tabular}

NT $=$ não testada 
Diversas técnicas vêm sendo utilizadas para determinar a atividade antioxidante in vitro, permitindo selecionar substâncias potencialmente ativas na prevenção de doenças degenerativas. ${ }^{33,34}$ Dentre elas destaca-se o método de seqüestro de radicais pelo DPPH, que se baseia na transferência de elétrons de um composto antioxidante para um oxidante. ${ }^{27}$

A Tabela 2 mostra os resultados obtidos no ensaio antioxidante do extrato etanólico das raízes de T. toxicaria (TTRE) e das frações oriundas da partição líquido-líquido deste extrato. TTRE e suas frações hexano (TTREH), clorofórmio (TTREC) e acetato de etila (TTREA) apresentaram atividade próxima ao padrão $\mathrm{BHT}\left(\mathrm{CI}_{50} 0,177\right.$ $\mathrm{mg} / \mathrm{mL}$ ). Entretanto, a fração metanol (TTREM) revelou-se a mais ativa, apresentando $\mathrm{CI}_{50} 0,089 \mathrm{mg} / \mathrm{mL}$ e resultado superior ao BHT. Dentre as substâncias isoladas, a obovatina (2) foi a única obtida em quantidade suficiente para permitir a avaliação de tal atividade e apresentou poder antioxidante inferior aos dos extratos testados $\left(\mathrm{CI}_{50}\right.$ $3,370 \mathrm{mg} / \mathrm{mL}$ ). Normalmente os compostos flavonóidicos, descritos como potenciais antioxidantes, ${ }^{35}$ são obtidos em maior quantidade nos extratos mais polares (e.g. metanólicos e aquosos $)^{36,37}$ o que corrobora com a maior atividade da fração metánolica em relação às demais frações. A abundância de substâncias fenólicas do tipo flavonóide no gênero Tephrosia ${ }^{38}$ pode justificar a atividade observada.

Tabela 2. Resultados de avaliação antioxidante de diferentes extratos e frações de T. toxicaria utilizando o DPPH como radical livre

\begin{tabular}{lc}
\hline Fração & CI $_{50}(\mu \mathrm{g} / \mathrm{mL})$ \\
\hline TTRE & 276 \\
TTREH & 264 \\
TTREC & 343 \\
TTREA & 218 \\
TTREM & 89 \\
\hline Substância isolada & $\mathrm{CI}_{50}(\mu \mathrm{g} / \mathrm{mL})$ \\
\hline Obovatina (2) & 3.370 \\
\hline Padrão & $\mathrm{CI}_{50}(\mu \mathrm{g} / \mathrm{mL})$ \\
\hline Vitamina C & 25 \\
Trolox & 17 \\
BHT & 177 \\
\hline
\end{tabular}

\section{CONCLUSÃO}

O estudo químico de Tephrosia toxicaria Pers. permitiu o isolamento de cinco flavonóides conhecidos: iso-obovatina (1), obovatina (2), 6a, 12adesidro- $\beta$-toxicarol (3), 6a, 12a-desidro- $\alpha$-toxicarol (4) e $\alpha$-toxicarol (5), confirmando o perfil quimiotaxonômico do gênero Tephrosia. Observouse ainda a presença de compostos contendo o sistema dimetilcromeno, corroborando o descrito na literatura. ${ }^{39-42}$ Os resultados dos extratos e substâncias de T. toxicaria nos ensaios de atividade larvicida contra o mosquito Aedes aegypti reforçam a utilização desta espécie como uma fonte de substâncias com potencial inseticida e justificam a sua utilização como defensivo agrícola, tornando-a uma alternativa ecologicamente viável para a erradicação ou controle da dengue.

Os ensaios de atividade antioxidante sugerem a espécie T. toxicaria como uma fonte promissora de agentes que podem ser utilizados contra o envelhecimento celular e o stress oxidativo.

\section{AGRADECIMENTOS}

Ao CNPq, CAPES, PRONEX, FUNCAP e FAPERJ pelas bolsas e auxílios financeiros concedidos, ao Centro Nordestino de Aplicação e
Uso de Ressonância Magnética Nuclear (CENAUREMN), Programa de Pós-graduação em Química Orgânica, Departamento de Química Orgânica e Inorgânica, Centro de Ciências, Universidade Federal do Ceará - Fortaleza - CE pelos espectros 1D e 2D de RMN ${ }^{1} \mathrm{H}$ e ${ }^{13} \mathrm{C}$ e ao Prof. E. P. Nunes, UFC, pela identificação do material vegetal.

\section{REFERÊNCIAS}

1. Sinha, B.; Natu, A. A.; Nanavati, D. D.; Phytochemistry 1982, 21, 1468.

2. Cabizza, M.; Angioni, A.; Melis, M.; Cabras, M.; Tuberoso, C. V.; Cabras, P.; J. Agr. Food Chem. 2004, 52, 288.

3. Moring, S. E.; McChesney, J. D.; J. Assoc. Off. Anal. Chem. 1979, 62, 774.

4. Yenesew, A.; Kiplagat, J. T.; Derese, S.; Midiwo, J. O.; Kabaru, J. M.; Heydenreich, M.; Peter, M. G.; Phytochemistry 2006, 67, 988.

5. Saleem, M.; Ahmed, S.; Alam, A.; Sultana, S.; Pharm. Res. 2001, 43, 135.

6. Andel, T. V.; Econ. Botany 2000, 54, 500.

7. Corrêa, M. P.; Dicionário de plantas úteis do Brasil e das exóticas cultivadas, Instituto Brasileiro de Desenvolvimento Florestal, Rio de Janeiro, 1974.

8. Clarck, E. P.; J. Am. Chem. Soc. 1930, 5, 2461.

9. Jang, D. S.; Park, E. J.; Kang, Y.; Hawthorne, E.; Vigo, J. S.; Grahan, J. G.; Cabieses, F.; Fong, H. H. S.; Mehta, R. G.; Pezzuto, J. M.; Kinghorn, A. D.; J. Nat. Prod. 2003, 66, 1166.

10. Ribeiro, W. H. F.; Vasconcelos, J. N.; Arriaga, A. M. C.; Oliveira, M. C. F.; Andrade-Neto, M.; Lemos, T. L. G.; Santiago, G. M. P.; Nascimento, R. F.; Mafezoli, J.; Nat. Prod. Comm. 2006, 1, 391.

11. Shaalan, E. A.; Canyon, D.; Younes, M. W. F.; Abdel-Wahab, H.; Mansour, A.; Environ. Int. 2005, 31, 1149.

12. Mendonça, F. A. C.; Silva, K. F. S.; Santos, K. K.; Júnior, K. A. L. R.; Sant'Ana, A. E. G.; Fitoterapia 2005, 76, 629.

13. Luna, J. S.; Santos, A. F.; Lima, M. R. F.; Omena, M. C.; Mendonça, F. A. C.; Bieber, L. W.; Sant'Ana, A. E. G.; J. Ethnopharmacol. 2005, 97 , 199.

14. Santos, R. P.; Nunes, E. P.; Nascimento, R. F.; Santiago, G. M. P.; Menezes, G. H. A.; Silveira, E. R.; Pessoa, O. D. L.; J. Braz. Chem. Soc. 2006, 17,1027

15. Prajapati, V.; Tripathi, A. K.; Aggrarwal, K. K.; Khanuja, S. P. S.; Biores. Technol. 2005, 96, 1749.

16. Ciccia , G.; Coussio, J.; Mongelli, E.; J. Ethnopharmacol. 2000, 72, 185.

17. Callaway, E.; Nature 2007, 448, 16.

18. Hugon, J.; Hugon, F.; Esclaire, F.; Lesort, M.; Diop, A. G.; Brain Research 1996, 707, 288.

19. Das, N. P.; Pereira, T. A.; J. Am. Oil Chem. Soc. 1990, 67, 255.

20. Vellosa, J. C. R.; Khalil, M. N.; Formenton, V. A. F.; Ximenes, V. F.; Fonseca, L. M.; Furlan, M.; Brunetti, I. L.; Oliveira, O. M. M. F.; Fitoterapia 2006, 77, 243.

21. Abdelwahed, A.; Bouhlel, I.; Skandrani, I.; Valenti, K.; Kadri, M.; Guiraud, P.; Steiman, R.; Mariotte, A.; Ghedira, K.; Laporte, F.; DijouxFranca, M.; Chekir-Ghedira, L.; Chem-Biol. Interact. 2007, 165, 1.

22. Cetinus, S. A.; Goze, I.; Saraç, B.; Vural, N.; Fitoterapia 2007, 78, 129.

23. Arriaga, A. M. C.; Magalhães, F. E. A; Feitosa, E. M. A., Malcher, G. T.; Andrade- Neto, M.; Nascimento, R. F.; J. Essent. Oil Res. 2005, 17, 451.

24. Gadelha, D. P.; Toda, A. T.; Rev. Brasil. Malariol. D. Trop. 1985, 37, 29.

25. Oliveira, M. F.; Lemos, T. L. G.; Matos, M. C.; Segundo, T. A.; Santiago, G. M. P.; Braz-Filho, R.; An. Acad. Bras. Cienc. 2002, 74, 211.

26. Cheng, S.; Chang, H.; Chang, S.; Tsai, K.; Chen, W.; Biores. Technol. 
2003, 89, 99 .

27. Lemos, T. L. G.; Machado, L. L.; Souza, J. S. N.; Fonseca, A. M.; Maia, J. L.; Pessoa, O. D. L.; Fitoterapia 2006, 77, 443.

28. de Oliveira, P. R. N.; Testa, G.; Sena, S. B.; Costa, W. F.; Sarragiotto, M. H.; Santin, S. M. O.; Quim. Nova 2008, 31, 755.

29. Argáez, R. B.; Diaz, M. E. P.; Waterman, P. G.; Rodriguez, L. M. P.; J. Braz. Chem. Soc. 2005, 16, 1078.

30. Andrei, C. C.; Ferreira, D. T.; Faccione, M.; Moraes, L. A. B.; Carvalho, M. G.; Braz-Filho, R.; Phytochemistry 2000, 55, 799.

31. Andrei, C. C.; Vieira, P. C.; Fernandes, J. B.; Silva, M. F. D. F.; Fo, E. R.; Phytochemistry 1997, 46, 1081.

32. Yenesew, A.; Derese, S.; Midiwo, J. O.; Heydenreich, M.; Peter, M. G.; Pest Manag. Sci. 2003, 59, 1159.

33. Tsai, H.; Chang, S. K. C.; Chang, S.; J. Agric. Food Chem. 2007, 55, 2867.
34. Elmastas, M.; Osildak, O.; Turkekul, I.; Temur, N.; J. Food Comp. Anal. 2007, 20, 337.

35. Banerjee, A.; Dasgrupta, N.; De, B.; Food Chem. 2005, 90, 727.

36. Kumaran, A.; Karunakaran, R. J.; Fitoterapia 2007, 78, 46.

37. Jayaprakasha, G. K.; Negi, P. S.; Jena, B. S.; Rao, J. M.; J. Food Comp. Anal. 2007, 20, 330.

38. Lima, J. Q.; Dissertação de Mestrado, Universidade Federal do Ceará, Brasil, 2005

39. Tarus, P. K.; Machocho, A. K.; Lang'At-Thoruwa, C. C.; Chhabra, S. C.; Phytochemistry 2002, 60, 375.

40. Rao, E. V.; Prasad, Y. R.; Phytochemistry 1993, 32, 183.

41. Dagne, E.; Dinku, B.; Gray, A. I.; Waterman, P. G.; Phytochemistry 1988, 27, 1503.

42. Venkataratnam, G.; Rao, E. V.; Vilain, C.; Phytochemistry 1986, 25, 1507. 